\title{
Software for Emulating the Sampled Values Transmission in Accordance with IEC 61850 Standard
}

\author{
Pavel F. Baranov, Sergey V. Muravyov, Almaz O. Sulaymanov, Lyudmila I. Khudonogova \\ Department of Computer-aided Measurement Systems and Metrology \\ National Research Tomsk Polytechnic University \\ Tomsk, Russian Federation \\ e-mail: [bpf, muravyov, sao, likhud] @tpu.ru
}

\begin{abstract}
At present there is an exigency in both software and hardware for emulation of measured sampled values transmission of electrical power parameters in order to test the digital substation equipment. Necessary software emulators implementing full functionality described in the standard IEC 61850 are still not exist. In the paper the software for emulating the transmission of measured sampled values in accordance with IEC 61850-9-2 and IEC 61850-9-2 LE is presented. It emulates transmission of sampled values at 80 or 256 samples per period for phase and neutral line sinusoidal currents and voltages and testing of digital substation equipment.
\end{abstract}

Keywords-sample values; power system simulation; smart power grids; IEC standards; protocols; digital substation

\section{INTRODUCTION}

Current trends in the electric power industry are development of digital substations in the framework of intelligent distributed networks (Smart Grid) [1]. The digital substation is a collection of intelligent electronic devises for electric power fiscal accounting, relay protection and automation and recording emergency events [2].

The International Electrotechnical Commission (IEC) published the standard IEC 61850 "Communication Networks and Systems in Substations" that regulates a design process of digital substations [3]. The particular standard IEC 61850-9-2 [4] describes the transmission protocol of measured sampled values of non-conventional instrument transformers in digital data networks.

Nowadays, there is an exigency in both software and hardware for emulation of measured sampled values transmission of electrical power parameters. These tools must be used to test the digital substation equipment in the course of its development and installation.

Many organizations are working in this area [5-9], however software emulators implementing full functionality described in the standard are still not exist.
This paper is devoted to a software development for emulating the transmission of measured sampled values in accordance with IEC 61850-9-2 and IEC 61850-9-2 LE (Implementation Guidelines for Digital Interface to Instrument Transformers) [10].

\section{Generating SAMPled VAlues Packet}

The standard IEC 61850 defines five types of communication services:

- $\quad$ Sampled Values (SV); (GOOSE);

Generic Object Oriented Substation Event

- $\quad$ Time synchronization (Time Sync);

- Manufacturing Messaging Specification (MMS);

- Generic Substation Status Event (GSSE).

SV, GOOSE and GSSE are time-critical, that is why they are mapped directly to Data Link layer of OSI-7 reference model that allows to reduce protocol overhead and hence to increase data transmission rate. Time Sync and MMS are transmitted with use of UDP and TCP/IP protocols respectively.

To transmit SV, GOOSE and GSSE the standard [4] defines a standardized Ethernet frame. The frame contains the field APDU (Application Protocol Data Unit) which includes the SV data. The APDU field structure for 256 samples per period is shown in Fig. 1.

APDU is transmitted in a format described by the triplet TLV (Type, Length and Value). Each of the fields T, L and $\mathrm{V}$ is a series of octets (bytes). The value $\mathrm{V}$, in its turn, can be the triplet TLV. Information of 256 sample values for one period of measured signals is transmitted by 32 packets, each packet includes 8 sample values.

The remainder of this section describes a composition of the APDU fields.

SavPdu field is the APDU starting one. The field length is 4 bytes: first byte is the field label, $0 \times 60$; second byte is 0x82, next two bytes hold the APDU length value. 


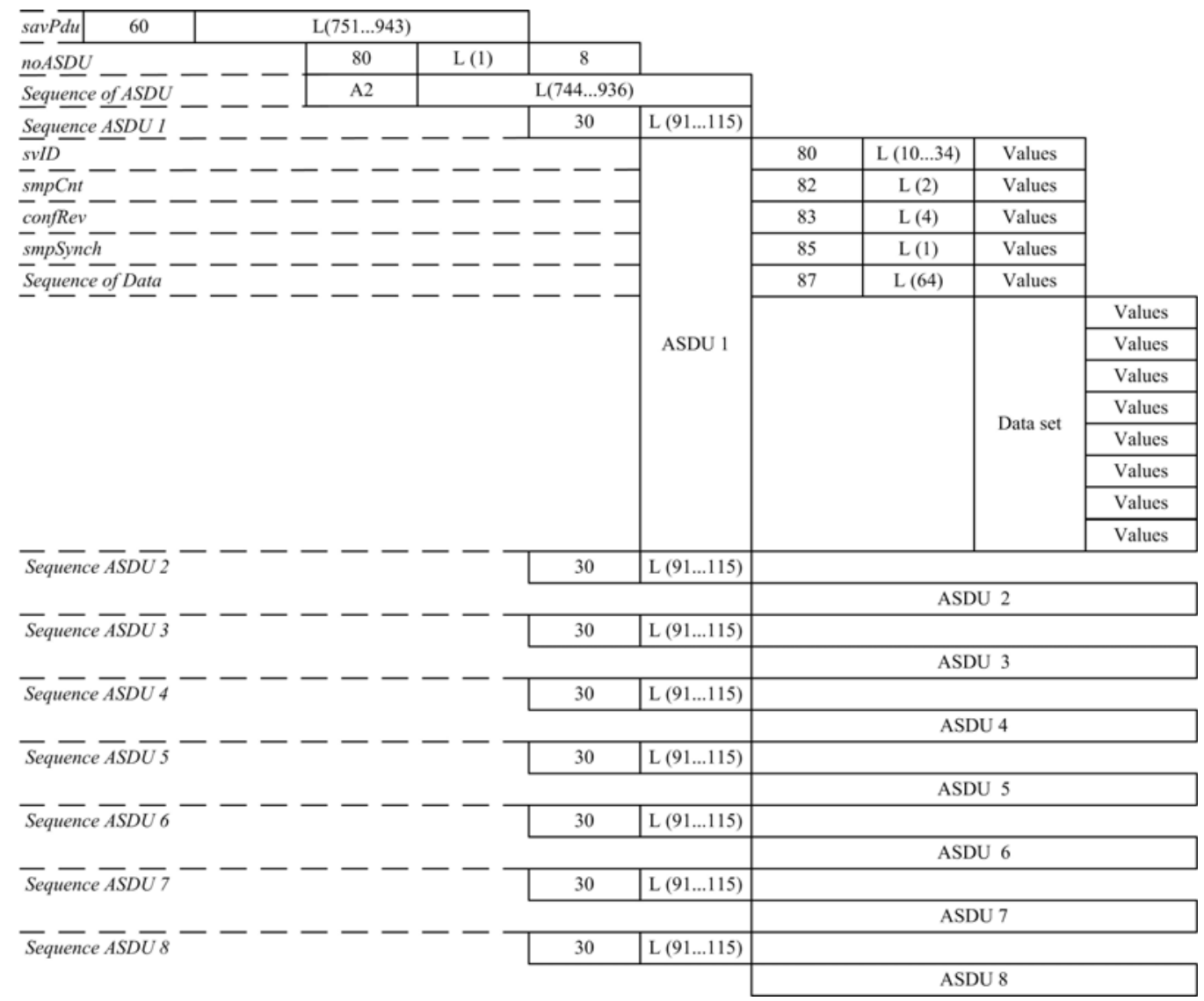

Figure 1. APDU structure for 256 samples per period

NoASDU field is the ASDU number. The field length is 3 bytes: first byte stores the field label equal to $0 \times 80$; second byte holds the value length equal to $0 \mathrm{x} 01$; third byte is equal to $0 \mathrm{x} 08$.

Sequence of ASDU field is all ASDUs starting part. The field length is 4 bytes: first byte is the field label, 0xA2; second byte is $0 \times 82$, next two bytes hold ASDUs length value.

Sequence ASDU 1 field is an identifier of the ASDU 1 start. The field length is 2 bytes: first byte is the field label equal to $0 \times 30$; second byte is ASDU 1 length.

SVID field is a name of the SV packet. The field length is from 21 to 69 bytes. First byte is the field label, 0x80; second byte hold SvID length value; next from 10 to 34 bytes are the value.

SmpCnt field is the sample number. The field length is 4 bytes: first byte is the field label, $0 \times 82$; second byte holds the value length equal to $0 \mathrm{x} 02$; next two bytes hold sample number.

ConfRev field is the configuration number. The field length is 6 bytes: first byte is the field label, 0x83; second byte holds the value length equal to $0 \times 04$; next four bytes hold configuration number value.

SmpSynch field is the label of the synchronization. The field length is 3 bytes: first byte is the field label, $0 \times 85$; second byte holds the value length equal to $0 \mathrm{x} 01$; third byte is synchronization value. The value is $0 \mathrm{x} 00$ for not synchronized sampled values, 0x01 for local synchronized sampled values and 0x02 for global synchronized sampled values.

Sequence of Data field is sequence of measured sampled values. The field length is 66 bytes: first byte is the field label, 0x87; second byte holds the value length equal to $0 \times 40$, next 40 bytes hold sequence of measured sampled values.

Sequence of Data field contains information about the sampled values of currents and voltages in A, B, C phases and neutral $\mathrm{N}$ transmission lines. Each measured value is represented by 8-byte hexadecimal code. An example of frame composition for one sampled value of current is shown in Fig. 2.

InnxTCTR1.Amp.instMag.i field is an amplitude value of sampled current, where $\mathrm{x}$ is $\mathrm{A}, \mathrm{B}$ or $\mathrm{C}$ phase. The scale factor is $1 \mathrm{bit}=1 \mathrm{~mA}$. The field length is 4 bytes.

InnxTCTR1.Amp.q field is an additional information about of sampled current, where $\mathrm{x}$ is $\mathrm{A}, \mathrm{B}$ or $\mathrm{C}$ phase. The field length is 2 bytes. The default value is equal to $0 \mathrm{x} 0000$.

Der field is an identifier of the derived current sampled value. The field length is 1 bit. The field indicates whether the value is measured ( $0 \mathrm{~b} 0)$ or calculated $(0 \mathrm{~b} 1)$. 


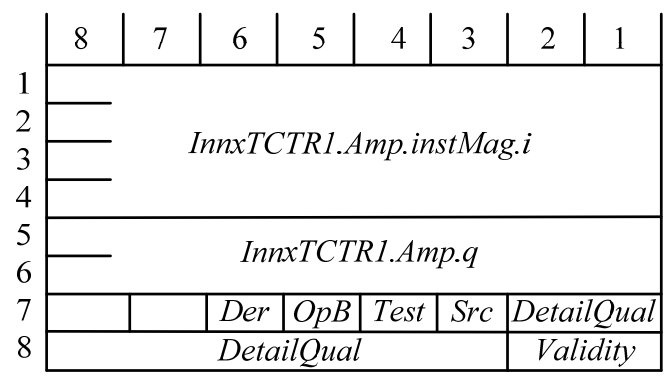

Figure 2. An example of frame format for one current sampled value

$O p B$ field identifies a mode of information blocking. The field length is 1 bit. This identifier should be set into 1 if further update of the value has been blocked. If this identifier is set then the identifier oldData in the field detailQual should also be set into 1 .

Test field is identifier of a mode functioning (working or testing). The field length is 1 bit. Field is used to classify the current value. If the mode is testing (0b1), then the value cannot be used for fiscal accounting.

Src field is an identifier of the source. The field length is 1 bit. Field gives information related to the origin of a value. The value can be acquired from the process $(0 \mathrm{~b} 0)$ or be a substituted (or calculated) value (0b1).

DetailQual field is an additional identifier of the data quality. The length field is 1 byte. The flags of the DetailQual are described in IEC 61850 7-3 [11].

Validity field is a data quality identifier and can be "good", "questionable" or "invalid". The length of the field is 2 bits. The default value is "good" $(0 \mathrm{~b} 00)$.

If, in the field DetailQual, there is at least one of the flags set into 1 , the field of validity must be set to the value "invalid" (0b01) or "questionable" (0b11).

If amplitudes of current and / or voltage are not measured, they broadcast in the network with "zero" values. In this case, validity must be set to "invalid" for these values.

\section{SOFTWARE FOR EMULATING TRANSMISSION OF SAMPLED VALUES}

Special software, virtual instrument (VI) "IED Emulator", was developed in the graphical programming environment LabVIEW for emulating the transmission of obtained sampled values and testing the digital substation equipment. The front panel of the developed virtual instrument is shown in Fig. 3.

The VI allows to select a network adapter, which is used for sampled values transmission, configure transmission parameters, namely: Destination address, TPID, User priority, CFI, VID, svID, reserved 1, reserved 2, smpSynch, and confRev - and emulate the measured sampled values transmission at 80 or 256 samples per period for phase and neutral line sinusoidal currents and voltages.

The VI also allows to change amplitude and spectral components of signals, the flags of DetailQual and synchronization fields immediately in the course of emulation. It provides an opportunity to test substation equipment in accordance with transmission information.

For operation with Data Link layer of OSI-7 reference model, special dynamic-link library (DLL) was developed using $\mathrm{C}++$ language. The DLL uses the library WinPcap (Windows Packet Capture).

\section{IV. “IED EMULATOR” VERIFICATION}

For experimental verification of the virtual instrument "IED Emulator" a network analyzer Wireshark was used. Wireshark supports data protocols of the IEC 61850 standard and allows to record relevant traffic.

Software SVScout was used for visual analysis of the developed VI functioning. SVScout subscribes to the SV streams with 80 samples per period from one or multiple intelligent electronic devices and displays the waveforms of the primary voltages and currents in an oscilloscope view [12].

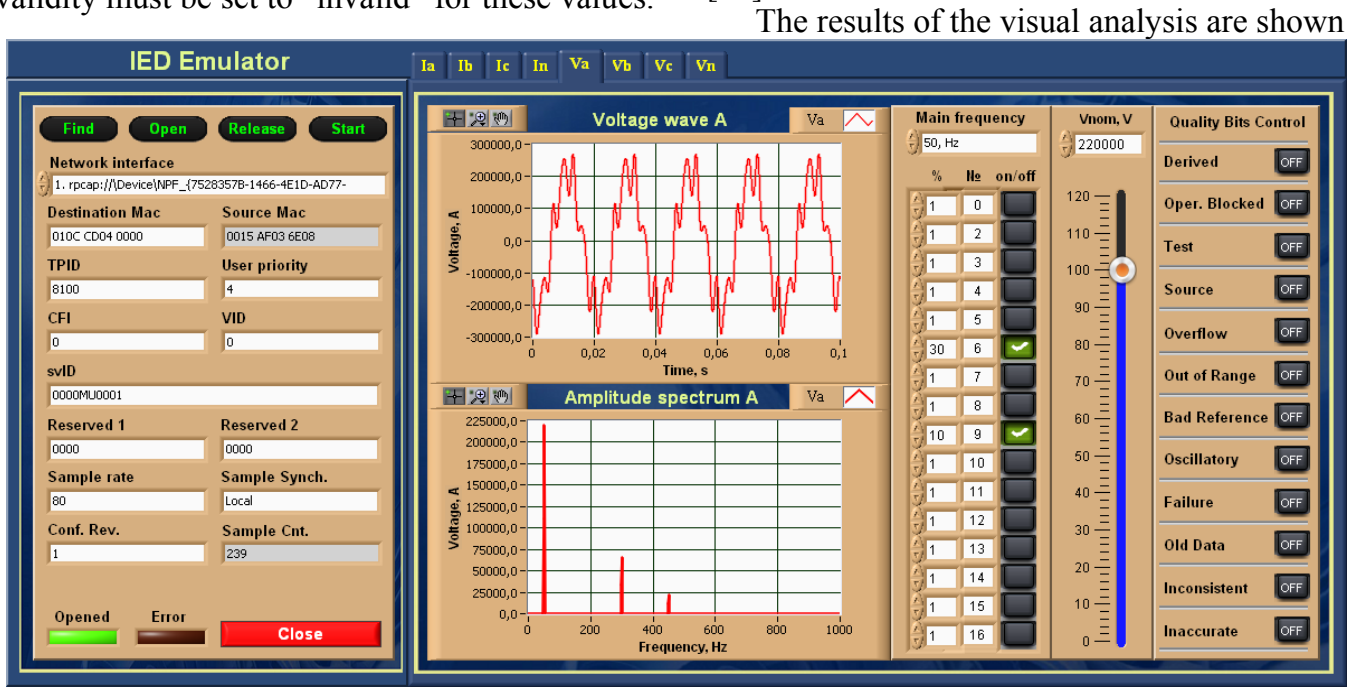

Figure 3. The front panel of the virtual instrument "IED Emulator" 


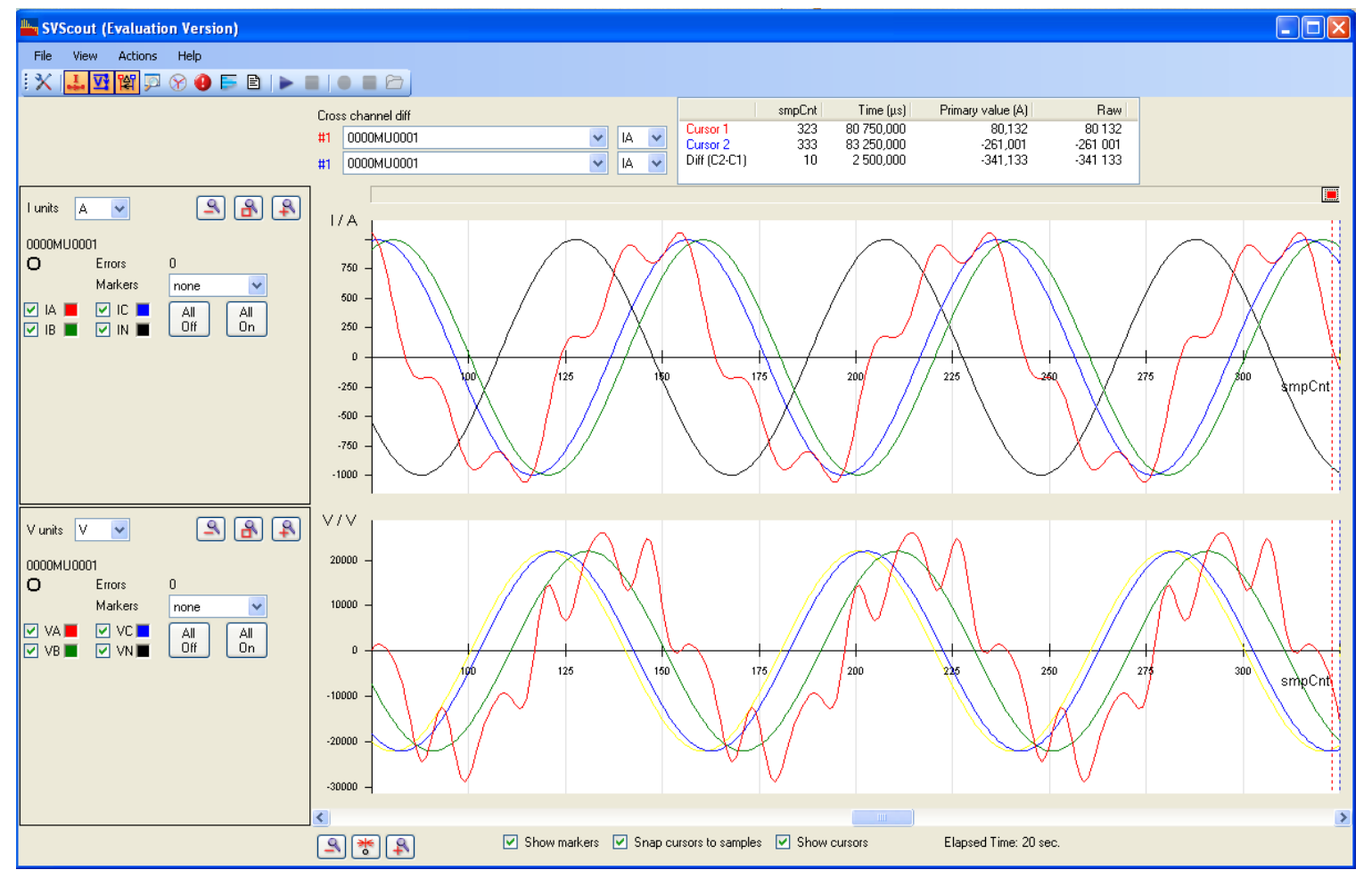

Figure 4. Results of the visual analysis

The verification using two independent programs has resulted in the following: data packet is generated and transmitted without errors; the sampled values data are uniquely decoded; the time intervals between transmitted packets do not exceed $120 \mu \mathrm{s}$.

\section{CONCLUSIONS}

1. The special software, "IED Emulator" has been developed for emulating transmission of sampled values at 80 or 256 samples per period for phase and neutral line sinusoidal currents and voltages and testing of digital substation equipment.

2. The verification of the software operation using two independent programs shows that data packet is generated and transmitted without errors and can be applied for the equipment conformance testing to the requirements of the standard IEC 61850 in the part of receiving and decoding sampled values.

3. The developed software can be used for hardware and software validation of the electric power measurement instruments at digital substations.

The research carried out in the under the federal target program "Research and development on priority directions of scientific-technological complex of Russia for 20072013." (Contract 14.516.12.0009).

\section{REFERENCES}

[1] N. Higgins, V. Vyatkin, N.C. Nair, K. Schwarz, "Concept for intelligent distributed power system automation with IEC 61850 and IEC 61499," in Systems, Man and Cybernetics, 2008. SMC 2008. IEEE International Conference on, 12-15 Oct. 2008, pp. 36-41
[2] J. McGhee, M. Goraj, "Smart High Voltage Substation Based on IEC 61850 Process Bus and IEEE 1588 Time Synchronization," in Smart Grid Communications (SmartGridComm), 2010 First IEEE International Conference on, 4-6 Oct. 2010, pp.489-494

[3] IEC 61850-1: Communication networks and systems in substations Part 1: Introduction and overview, 1st ed. IEC, 2003

[4] IEC 61850-9-2: Communication networks and systems in substations - Part 9-2: Specific Communication Service Mapping (SCSM) Sampled values over ISO/IEC 8802-3, 1st ed. IEC, 2004

[5] J.W. Konka, C.M. Arthur, F.J. Garcia, R.C.Atkinson, "Traffic generation of IEC 61850 sampled values," in Smart Grid Modeling and Simulation (SGMS), 2011 IEEE First International Workshop on, 17-17 Oct. 2011, pp. 43-48

[6] R. Kuffel, D. Ouellette, P. Forsyth, "Real time simulation and testing using IEC 61850," in Modern Electric Power Systems (MEPS), 2010 Proceedings of the International Symposium, 20-22 Sept. 2010, pp. $1-8$

[7] Triangle MicroWorks - Protocol Solutions For IEC 61850. Available: $\mathrm{http} / / / \mathrm{www}$.trianglemicroworks.com/ProductPage.aspx

[8] OMICRON - Products. Available: http://www.omicron.at/en/ products/pro/

[9] TEKVEL - IEC 61850. Available: http://tekvel.ru

[10] IEC 61850-9-2 LE: Implementation Guideline for Digital Interface to Instrument Transformers Using IEC 61850-9-2. Available: http://www.ucainter-national.org

[11] IEC 61850-7-3: Communication networks and systems in substations - Part 7-3: Basic communication structure for substation and feeder equipment - Common data classes 3, 1st ed. IEC, 2003

[12] SVScout - Software Tool for Visualizing IEC 61850 Sampled Values., Available: http://www.omicron.at/en/products/pro/communication-protocols/ svscout/ 